15 Leertouwer TC, Pattynama PMT, van den Berg-Huysmans A. Incidental renal artery stenosis in peripheral vascular disease: a case for treatment? Kidney Int 2001;59: $1480-3$.

\section{SELF-ASSESSMENT QUESTIONNAIRE}

\section{Renal Medicine}

Ten self-assessment questions (SAQs) based on the published articles will appear at the end of each CME specialty featured in Clinical Medicine. The questions have been validated for the purpose of CME by independent experts. Two (2) CME credits will be awarded to those achieving $80 \%$ correct answers. This opportunity is open only to RCP Fellows and Collegiate Members in the UK who are registered for CME*.

A loose leaf answer sheet is enclosed, which will be marked electronically at the Royal College of Physicians. Answer sheets must be returned by 21 December 2003 to:

CME Department (SAQs), Royal College of Physicians,

11 St Andrews Place, London NW1 4LE.

Overseas members only can fax their answers to 02074874156

Correct answers will be published in the next issue of Clinical Medicine.

*Further details on CME are available from the CME department at the Royal College of Physicians (address above or telephone 02079351174 extension 306 or 309).

\section{Guidelines on completing the answer sheet}

Your completed answer sheet will be scanned to enable a quick and accurate analysis of results. To aid this process, please keep the following in mind:

1 Please print your GMC Number firmly and neatly

2 Only write in allocated areas on the form

3 Only use pens with black or dark blue ink

4 For optimum accuracy, ensure printed numbers avoid contact with box edges

5 Please shade circles like this: Not like this: $)$

6 Please mark any mistakes made like this: $\lambda$

7 Please do not mark any of the black squares on the corners of each page

8 Please fill in your full name and address on the back of the answer sheet in the space provided; this will be used to mail the form back to you after marking.
A 35-year-old woman, who is known to have autosomal dominant polycystic kidney disease (ADPKD) and mild hypertension, comes to the clinic with her eight-year-old daughter. She is aware of the possibility that she has passed ADPKD on to her daughter. The daughter is asymptomatic, but the mother wonders if she needs to be screened. The mother has two other children, aged five and three years. Which of the following statements are true and which are false?

(a) A DNA sample (venous blood or buccal scraping) should be obtained from the daughter and sent for mutational analysis

(b) The daughter should be thoroughly examined, including blood pressure (BP) and urinalysis; if no abnormalities are found and the kidneys cannot be palpated, the mother can be reassured that her child is not affected

(c) An ultrasound scan should be arranged; the diagnosis of ADPKD is confirmed even if this shows only one cyst in one kidney 
(d) An ultrasound scan should be arranged; if this confirms the diagnosis, the other children should also be offered screening

(e) The mother should be offered genetic counselling and advised not to have her children screened unless there is a specific clinical indication

2 A 45-year-old man is found to have dipstick positive

haematuria $2+$ at his general practitioner (GP) surgery during a new patient check. There is no proteinuria and no previous record of urinalysis. On repeated measurements, his resting seated BP averages $145 / 95 \mathrm{mmHg}$ in both arms. $\mathrm{His}$ body mass index is $35 \mathrm{~kg} / \mathrm{m}^{2}$. Flexible cystoscopy and renal ultrasound scanning show no urological abnormality. Estimated glomerular filtration rate (GFR), based on age, gender, weight and serum creatinine $(\mathrm{SCr})$, is $80 \mathrm{ml} / \mathrm{min}$. Which of the following statements are true and which are false?

(a) There is a $50 \%$ chance that a renal biopsy would show glomerular disease

(b) If immunoglobulin A nephropathy is present on renal biopsy, immunosuppressive therapy aimed at suppressing glomerulonephritis will result in improved BP control

(c) The risk of major complications from renal biopsy is under one in 200

(d) There is a high likelihood of progressive loss of renal function and eventual renal failure

(e) He should be advised to return to his GP annually for dipstick testing for proteinuria and measurement of $\mathrm{SCr}$

3 A 70-year-old woman presents with fatigue and exertional dyspnoea, and is found to have a normochromic, normocytic anaemia and a $\mathrm{SCr}$ of $350 \mu \mathrm{mol} / \mathrm{l}$, giving an estimated GFR of $35 \mathrm{ml} / \mathrm{min}$. Which of the following statements are true and which are false?

(a) A negative dipstick test for proteinuria makes it extremely unlikely that a renal biopsy will disclose treatable renal disease

(b) Reduced renal bipolar length on ultrasound scanning increases the probability that a biopsy will show nonspecific and irreversible histological changes

(c) A single kidney on ultrasound scanning would present an absolute contraindication to renal biopsy

(d) If she is found to have myeloma on serum electrophoresis and assay for urinary light chains, a renal biopsy would be necessary to decide the most appropriate treatment

(e) A protein:creatinine ratio of $300 \mathrm{mg} / \mathrm{mmol}$ on a random urine sample would be an indication for an angiotensin-converting enzyme inhibitor (ACEI), angiotensin receptor blocker or both, aiming for a target BP of $120 / 75 \mathrm{mmHg}$

4 A 36-year-old woman with nephrotic syndrome but normal renal function has been treated with a combination of intravenous (iv) loop diuretics in high dosage and oral spironolactone. She is restricted to a dietary salt intake of $60 \mathrm{mmol} /$ day and a water intake of $2.5 \mathrm{l} /$ day. She has developed a hyponatraemic (serum [sodium]) $125 \mathrm{mmol} / \mathrm{l}$ ) metabolic alkalosis (total [bicarbonate] $34 \mathrm{mmol} / \mathrm{l})$. Although still oedematous, her serum [urea] has increased dramatically and she has developed gout. Which of the following statements are true and which are false?

(a) A thiazide diuretic should be added to the above regimen

(b) The hyponatraemia is most likely due to spironolactone

(c) The addition of cimetidine will prevent further hyperuricaemia and gout

(d) Her prescription for dietary salt and water intake is appropriate (e) She has recently had a baby; breast-feeding should be discouraged

5 A 22-year-old man with nephrotic syndrome has achieved euvolaemia on combination treatment with oral bumetanide $5 \mathrm{mg}$ and oral bendroflumethiazide $2.5 \mathrm{mg}$. Serum [potassium] is $2.9 \mathrm{mmol} / \mathrm{l}$ one week later. He has also developed an unpleasant skin rash which was present with diuretic agent use in the past. Which of the following statements are true and which are false?

(a) Hypokalaemia is an unusual feature of this drug combination

(b) Changing his drug treatment to furosemide and metolazone may not improve the rash

(c) He probably requires an iv infusion of albumin

(d) He probably also has hypocalcaemia

(e) He can abandon dietary salt intake restriction now that euvolaemia has been achieved

6 A 65-year-old woman who was found to have type 2 diabetes mellitus eight years ago presents with increasing fatigue and breathlessness. She weighs $50 \mathrm{~kg}$. Her BP is $160 / 95 \mathrm{mmHg}$. Initial investigations reveal the following results: $\mathrm{SCr}$ $125 \mu \mathrm{mol} / \mathrm{l}$, haemoglobin (Hb) $9.8 \mathrm{~g} / \mathrm{dl}$, with urine reagent strip testing showed dipstick protein $2+$, blood negative, glucose $2+$. Which of the following statements are true and which are false?

(a) Her anaemia is unlikely to be related to her renal function because her $\mathrm{SCr}$ is not significantly elevated

(b) Erythropoietin (EPO) therapy is contraindicated because she is hypertensive

(c) A myeloma screen should be performed

(d) An ECG is unlikely to show a significant abnormality 
(e) She should receive iv iron if her serum ferritin is below $150 \mu \mathrm{g} / \mathrm{l}$ and her transferrin saturation below $20 \%$.

7 A 75-year-old man with New York Heart Association class III heart failure and

SCr $250 \mu \mathrm{mol} / \mathrm{l}$ is found to have an $\mathrm{Hb}$ of $10.9 \mathrm{~g} / \mathrm{dl}$. Which of the following statements are true and which are false?

(a) His anaemia is an independent risk factor for death

(b) Correction of the anaemia is unlikely to improve his exercise tolerance

(c) His risk of being admitted to hospital can be substantially reduced by aiming for an $\mathrm{Hb}$ greater than $12 \mathrm{~g} / \mathrm{dl}$.

(d) He should receive EPO therapy iv as this is more efficient than the subcutaneous route

(e) Treatment of the anaemia will not influence the progression of his chronic kidney disease

8 A 67-year-old woman with tablet-treated diabetes mellitus, hypertension and a baseline $\mathrm{SCr}$ of $200 \mu \mathrm{mol} / \mathrm{l}$ is started on an ACEI. After three days of treatment, the $\mathrm{SCr}$ is $246 \mu \mathrm{mol} / \mathrm{l}$. Which of the following statements are true and which are false?

(a) ACEl must be stopped immediately

(b) ACEl can be continued with repeat $\mathrm{SCr}$ in one month

(c) Renal ultrasound scan is indicated, if no previous renal imaging

(d) Renal artery imaging is indicated

(e) If the rise in $\mathrm{SCr}$ is due to renal artery stenosis, it must be bilateral

9 A 78-year-old man with a long history of hypertension is found to have an SCr of $140 \mu \mathrm{mol} / \mathrm{I}$. Which of the following statements are true and which are false?

(a) A kidney and bladder ultrasound will exclude obstruction as the cause (b) An isotopic GFR should be performed to assess renal function more accurately

(c) This degree of elevation of $\mathrm{SCr}$ may be solely due to age-related decline in GFR

(d) Proteinuria greater than $2+$ is an indication to send urine to the laboratory for an albumin creatinine ratio

(e) Given his age, a BP of $160 / 90 \mathrm{mmHg}$ represents adequate control

10 A 79-year-old man of African-Caribbean ethnic origin has been known to have an $\mathrm{SCr}$ of 250-300 $\mu \mathrm{mol} / \mathrm{l}$ for some years, associated with hypertension and diet-controlled type 2 diabetes. Which of the following statements are true and which are false?

(a) Referral to a renal physician is unnecessary unless he develops symptoms of uraemia such as nausea, anorexia and pruritus

(b) African-Caribbean patients have a higher $\mathrm{SCr}$ for a given GFR

(c) His age means that he is not suitable for renal replacement therapy (dialysis or transplantation)

(d) He is unlikely to have diabetic nephropathy in view of his diet-controlled diabetes

SAQs and

answers - online for RCP Fellows and Members

From Volume 4 onwards, the SAQs in the CME sections of this journal can be answered online to achieve External CPD credits.

Look out for

further details in the January/February 2004 issue of Clinical Medicine (e) Hypertension is rarely a cause of renal disease in African-Caribbean patients

\section{CME Elderly Medicine SAQs}

\section{Answers to the CME SAQs published in Clinical Medicine September/October 2003}

$\begin{array}{llllllllll}\text { Q1 } & \text { Q2 } & \text { Q3 } & \text { Q4 } & \text { Q5 } & \text { Q6 } & \text { Q7 } & \text { Q8 } & \text { Q9 } & \text { Q10 } \\ \text { a) } F & \text { a) } F & \text { a) } F & \text { a) } F & \text { a) } F & \text { a) } T & \text { a) } F & \text { a) } T & \text { a) } F & \text { a) } T \\ \text { b) } F & \text { b) } F & \text { b) } T & \text { b) } T & \text { b) } F & \text { b) } F & \text { b) } T & \text { b) } T & \text { b) } T & \text { b) } F \\ \text { c) } T & \text { c) } F & \text { c) } T & \text { c) } T & \text { c) } T & \text { c) } T & \text { c) } F & \text { c) } F & \text { c) } F & \text { c) } F \\ \text { d) } T & \text { d) } T & \text { d) } F & \text { d) } T & \text { d) } F & \text { d) } F & \text { d) } F & \text { d) } F & \text { d) } T & \text { d) } F \\ \text { e) } T & \text { e) } T & \text { e) } F & \text { e) } F & \text { e) } F & \text { e) } F & \text { e) } F & \text { e) } T & \text { e) } F & \text { e) } T\end{array}$

